\title{
Plasma flows around magnetic obstacles in the solar wind
}

\author{
E. Romashets ${ }^{1,2,3}$ and S. Poedts ${ }^{1}$ \\ 1 Centre for Plasma Astrophysics and Leuven Mathematical Modelling and Computational Science Centre, K.U. Leuven, \\ 3001, Belgium \\ 2 Institute of Terrestrial, Magnetism, Ionosphere, and Radio Wave Propagation of Russian Academy of Sciences, Troitsk, \\ 142092, Russia \\ 3 Solar Observatory of Prairie View A\& M University, Prairie View, 77446, USA \\ e-mail: eromashets@pvamu.edu
}

Received 20 June 2006 / Accepted 13 September 2007

\begin{abstract}
Context. Recent numerical simulations and data analysis have shown that the area in front of magnetic clouds is very important from the point of view of its geo-efficiency. This area has very complicated magnetic and plasma structures. It is necessary to describe the plasma parameter distributions in the vicinity of magnetic clouds and other stable structures in the solar wind. Assuming that the magnetic field around the object is determined or measured, the velocity field is calculated from the frozen-in equation, while the density and pressure are given by explicit formulas expressing $P$ and $\rho$ as functions of only $\boldsymbol{B}$ and $\boldsymbol{V}$. An alternative method is to solve the full system of MHD equations numerically, but even in this case the analytical estimates determined here are also useful when formulating initial and boundary conditions.

Aims. The aim is to treat the region in front of interplanetary magnetic clouds in terms of analytical functions for a detailed consideration of general phenomena and also for particular phenomena of specific clouds.

Methods. First, the velocity and magnetic field distributions satisfying the boundary conditions and the frozen-in condition are determined. Next, the plasma density and pressure are calculated.

Results. The three-dimensional plasma parameter distributions are found for the general case of an inclined cylindrical cloud.
\end{abstract}

Key words. solar wind - Sun: magnetic fields - solar-terrestrial relations - Sun: coronal mass ejections (CMEs)

\section{Introduction}

Recent numerical simulations (Manchester et al. 2004; Chane et al. 2006) and data analysis (Owens et al. 2005) show that the area in front of magnetic clouds is very important from the point of view of its geo-efficiency. Moreover, this area has very complicated magnetic and plasma structures. Yet, the ability to treat it in terms of analytical functions would be necessary for a detailed consideration of general phenomena of magnetic clouds and their geo-efficiency, and also for studying particular phenomena of specific clouds in event studies.

The mathematical model of magnetohydrodynamics (MHD) provides a description of both the macroscopic behaviour of a plasma embedded in a magnetic field and the interaction between the plasma and the magnetic field. The single-fluid MHD equations, together with an appropriate geometry and boundary conditions, often provide a satisfactory model for describing the plasma dynamics, both in the laboratory and in space. Where necessary, additional assumptions or effects can be included or added to this model. For example, one such possibility is to assume flow incompressibility, defined by $\nabla \cdot \boldsymbol{V}=0$ (Farrugia et al. 1987; Erkaev et al. 1995). With this additional assumption, some of the MHD equations become independent of each other (they decouple), hence simplifying the system considerably. Even this reduced system is still not analytically solvable in general in full three-dimensional geometry. However, in the two-dimensional case, it follows from the frozen-in condition that, if the magnetic field has one or more stagnation points (where $\boldsymbol{V}=0$ ), then $\boldsymbol{V} \| \boldsymbol{B}$ in the entire volume. As a consequence, analytical solutions become feasible.

Another possible simplification is to assume that the magnetic field is current-free, i.e. $\nabla \times \boldsymbol{B}=0$. This assumption facilitates the solution of the equation of motion considerably, although it still does not enable the solution of the system in full three-dimensional geometry. Yet, the ability to treat the general case with an arbitrary angle between the velocity and the magnetic field far from an obstacle is very important for the mathematical modelling of disturbances in the solar wind. As a matter of fact, observations at $1 \mathrm{AU}$, as well as numerical, three-dimensional MHD simulations, show that the $z$ - and $y$-components of the velocity field almost always have negligible values in comparison with $V_{x}$. For the magnetic field vector $\boldsymbol{B}$, which lies as a rule within the $x y$-plane, the $x$ - and $y$-components are of comparable magnitude. Moreover, the magnetic field sometimes also has a strong $z$-component (Gosling \& McComas 1987; McComas \& Gosling 1988; Detman et al. 1991; Odstrcil \& Pizzo 1999). This problem can also be solved under the condition $\nabla \cdot V \neq 0$, with a pressure and density that are only functions of $\boldsymbol{B}$ and $\boldsymbol{V}$, while the magnetic field is determined by boundary conditions at infinity and at the obstacle surface; and the velocity is derived from the frozen-in condition.

The proposed model can serve as a tool for studying deflected flows around interplanetary magnetic clouds. This study is important for a better understanding of the quasi steady-state 
expansion of such clouds and for the derivation of the cloud's shape prior to arrival on the Earth's orbit, as these parameters determine the geo-efficiency of the cloud impact. Moreover, this approach to investigating deflected flows can help to improve the evaluation of the drag force acting on ICMEs because of the difference between the cloud's speed and the velocity of the ambient draping solar wind (Cargill 2004; Vršnak 2001). This force becomes smaller due to the decrease in the effective Newton's constant, which is now much smaller than unity.

In the next section we describe the model and the equations used. Section 3 then contains the results obtained, first for the case with $B_{z}=0$, and then for the more general case with $B_{z} \neq 0$. In Sect. 4 we discuss how the density and the plasma pressure can be determined from the equations once the velocity and the magnetic field is known. Finally, the conclusions are drawn in Sect. 5.

\section{Model, equations, and solution method}

We are interested in determining the plasma flow around a cylindrical object modelling a CME flux rope in the solar wind or in the solar corona. We are looking for a time-independent (steady) solution and we thus consider the equations of ideal magnetohydrodynamics (MHD) in the stationary form (cgs units), i.e.,

$\nabla \cdot \boldsymbol{B}=0$

$\nabla \times(\boldsymbol{V} \times \boldsymbol{B})=0$,

$\nabla \cdot(\rho \boldsymbol{V})=0$,

$\rho(\boldsymbol{V} \cdot \nabla) \boldsymbol{V}+\nabla\left(P+\frac{\boldsymbol{B}^{2}}{8 \pi}\right)=\frac{(\boldsymbol{B} \cdot \nabla) \boldsymbol{B}}{4 \pi}$,

$V \cdot \nabla\left(\frac{P}{\rho^{\gamma}}\right)=0$.

In Eqs. (1)-(5), $\boldsymbol{V}$ is the velocity of the plasma around the magnetic cloud in the reference frame of the magnetic cloud, $\boldsymbol{B}$ the magnetic field, $\rho$ the mass density, and $P=n k T$ the gas pressure. We assume $\gamma$ has the adiabatic value for an ideal gas, i.e. $\gamma=5 / 3$.

Observations showed that the temperature of the protons in the region around the magnetic clouds is much higher than that of the electrons (Farrugia et al. 1994). Hence, we may neglect the electron contribution to the temperature and treat the problem in the one-fluid theory. We also assume an isotropic pressure. In addition, the cloud is far enough from the Sun (say more than $0.3 \mathrm{AU}$ ), so the gravity force is not taken into account in our model. Moreover, the plasma flows are subsonic with respect to the cloud. Hence, the Mach and Alfvén Mach numbers are less than unity: $M<1, M_{\mathrm{a}}<1$. We model the plasma tube as a rigid, infinite cylindrical body embedded in the surrounding solar-wind magneto-plasma flow. We require that there are no magnetic and flow field components through the tube boundary.

It has been shown by numerical simulations that interplanetary coronal mass ejections (ICMEs) can be treated as cylinders with non-circular cross-sections (Riley et al. 2004; Riley \& Crooker 2004; Manchester et al. 2004; Owens et al. 2006). The approach adopted in this paper can be applied to non-circular cylindrical ICMEs as well. In that case, elliptical coordinates should be used (Romashets \& Vandas 2003). The study of deflected flow is important for determining the expansion of the cloud. The deflected flow forms an asymmetric pressure distribution around the rope, which is one of the reasons for its asymmetric expansion. Non-radial flows around 5 interplanetary clouds were studied in Owens \& Cargill (2004), who found that the averaged non-radial component of the velocity, caused by the draping effect, is of the order of $100 \mathrm{~km} \mathrm{~s}^{-1}$. Upstream flow deflections also provide a possible means of inferring the crosssectional shape and extent of ejecta.

Gravity is not considered in the present paper; that is, the model is only applicable beyond 0.3 AU. Moreover, since the cross-sectional elongation is increasing with distance from the Sun, we cannot apply the circular solution presented here beyond $0.7 \mathrm{AU}$.

We describe the plasma dynamics here in terms of a local cylindrical coordinate system $(r, \varphi, z)$ with the $z$-axis directed along the symmetry axis of the cylindrical flux rope. Usually, all these equations are solved simultaneously by means of a numerical procedure. Here, however, we follow another approach, by first selecting the magnetic field configuration satisfying the following boundary conditions:

1. the normal component of the magnetic field vanishes at the surface of the flux tube; and

2. far away from the flux tube, the magnetic field approaches its regular, undisturbed values, i.e. the values it would have without the insertion of the flux rope.

Of course, we also assume that the magnetic field is finite everywhere, and the electric current density is also supposed to be finite in the region under consideration. The number of magnetic field configurations satisfying these boundary conditions is limited, although there are several possible solutions. Below, we first derive a solution with $B_{z}=0$, and then a more general solution with a non-vanishing axial magnetic field component. Clearly, when there are additional constraints on the nature of the magnetic field, e.g. if it is known that the field is potential (current-free), then there is a unique solution satisfying these boundary conditions. However, although such additional assumptions simplify the solution considerably, a current-free magnetic field is not acceptable in most cases because, in general, the Lorentz force is substantial and plays an important role in the dynamics and evolution of a flux rope in the solar corona and the solar wind. We are thus looking for a solution with a finite (non-zero) electric current density. Clearly, there is a wide variety of possible current distributions. Among the many possibilities, we choose a natural current structure that is consistent with the observations and with the considered geometry. We can require, for example, that the currents are directed only along the $z$-axis.

Next, we derive the velocity field from the above-mentioned MHD equations and, in particular, Eq. (2). Finally, Eqs. (3)-(5) will yield the plasma density and plasma pressure consistent with the derived velocity field and the chosen magnetic field configuration.

\section{Solutions}

We first determine the velocity field for a simple magnetic field configuration without an axial component. Next, we derive the velocity for a more general case with a non-vanishing $z$-component of the magnetic field.

\subsection{The case $B_{z}=0$}

To find the corresponding frozen-in flow field in the case $B_{z}=0$, one can use the following sample field distribution in cylindrical 
coordinates $(r, \varphi, z)$ :

$B_{r}=B_{0}\left(1-\frac{r_{0}^{3}}{r^{3}}\right) \cos \varphi$,

$B_{\varphi}=-B_{0}\left(1+2 \frac{r_{0}^{3}}{r^{3}}\right) \sin \varphi$

$B_{z}=0$.

From the physical point of view, Eqs. (6)-(8) yield a magnetic field draping around a cylindrical cloud of radius $r_{0}$, which has "superconductive" boundary walls that prevent field lines from penetrating its interior. This magnetic field is not current-free. The corresponding electric current is directed along the $z$-axis and is both $r$ - and $\varphi$-dependent.

The frozen-in condition,

curl $\boldsymbol{V} \times \boldsymbol{B}=0$,

is mathematically equivalent to

$\boldsymbol{V} \times \boldsymbol{B}=\nabla F$,

where $F$ is a scalar function. By rewriting Eq. (10) we obtain, for the three components of this equation,

$-V_{z} B_{\varphi}=V_{z} B_{0}\left(1+2 \frac{r_{0}^{3}}{r^{3}}\right) \sin \varphi=\frac{\partial F}{\partial r}$,

$V_{z} B_{r}=V_{z} B_{0}\left(1-\frac{r_{0}^{3}}{r^{3}}\right) \cos \varphi=\frac{1}{r} \frac{\partial F}{\partial \varphi}$,

$V_{r} B_{\varphi}-V_{\varphi} B_{r}=\frac{\partial F}{\partial z}$

To apply the method of separation of variables, we assume

$F(r, \varphi, z)=R(r) \Phi(\varphi) Z(z)$,

and combining Eqs. (11) and (12), we obtain

$\frac{\Phi \frac{\mathrm{d} R}{\mathrm{~d} r}}{\left(1+2 \frac{r_{0}^{3}}{r^{3}}\right) \sin \varphi}=\frac{R \frac{\mathrm{d} \Phi}{\mathrm{d} \varphi}}{r\left(1-\frac{r_{0}^{3}}{r^{3}}\right) \cos \varphi}$

or, by separating the variables:

$\frac{r}{R} \frac{r^{3}-r_{0}^{3}}{r^{3}+2 r_{0}^{3}} \frac{\mathrm{d} R}{\mathrm{~d} r}=\frac{1}{\Phi} \frac{\sin \varphi}{\cos \varphi} \frac{\mathrm{d} \Phi}{\mathrm{d} \varphi}$.

Since the left-hand side (LHS) only depends on $r$ and the righthand side (RHS) only on $\varphi$, this equation can only be valid for all $r$ and all $\varphi$ when both the LHS and the RHS are constant. When choosing this (positive) constant as $k>0$, the equation for $\Phi$ reads

$\frac{\mathrm{d} \Phi}{\Phi}=k \frac{\cos \varphi \mathrm{d} \varphi}{\sin \varphi}$

and thus we obtain for $\Phi(\varphi)$

$\Phi=A \sin ^{k} \varphi$,

where, $A$ is an arbitrary integration constant.

Formally, any positive value of $k$ should give a mathematically correct solution for $\Phi$. However, not all these solutions make physical sense. High values of $k$ yield a very oscillatory function $\Phi(\varphi)$, which is inconsistent with the chosen magnetic field configuration (6)-(8). Therefore, we choose $k=1$. This means that the velocity distribution is smoothed, and we can no longer see the details. In principle, saving terms with $k>1$ can yield a finer structure, giving us

$\Phi=A \sin \varphi$.

Similarly, we obtain from Eq. (14) for $R(r)$ (putting the LHS again equal to the same constant $k$ )

$$
\begin{aligned}
\frac{\mathrm{d} R}{R} & =\frac{k}{r} \frac{r^{3}+2 r_{0}^{3}}{r^{3}-r_{0}^{3}} \mathrm{~d} r=\frac{k}{3} \frac{1}{r^{3} / r_{0}^{3}} \frac{r^{3} / r_{0}^{3}+2}{r^{3} / r_{0}^{3}-1} \mathrm{~d}\left(\frac{r^{3}}{r_{0}^{3}}\right) \\
& =\frac{k}{3}\left(\frac{3}{r^{3} / r_{0}^{3}-1}-\frac{2}{r^{3} / r_{0}^{3}}\right) \mathrm{d}\left(\frac{r^{3}}{r_{0}^{3}}\right) \\
& =\frac{k}{3} \mathrm{~d}\left\{3 \ln \left(r^{3} / r_{0}^{3}-1\right)-2 \ln \left(r^{2} / r_{0}^{2}\right)\right\} \\
& =\mathrm{d}\{\ln R+\text { const. }\} .
\end{aligned}
$$

Solving this equation we obtain

$R=C \frac{\left(r^{3} / r_{0}^{3}-1\right)^{k}}{\left(r / r_{0}\right)^{2 k}}$

where $C=$ const. For $k=1$, this solution reduces to

$R=C \frac{r^{3}-r_{0}^{3}}{r^{2} r_{0}}$

The function $Z(z)$, is not involved in the separation of variables in this case, because $B_{z}=0$. However, it must be chosen so that Eqs. (11)-(13) are satisfied. It is set here in the form

$Z=D\left(1+\alpha \sin \frac{z}{z_{0}}\right)$

where $D=$ const., $\alpha=$ const., and $z_{0}=$ const. Combining Eqs. (16), (18), and (20), the scalar function $F$ is, in the case $k=1$,

$F=B_{0} V_{0} \frac{r^{3}-r_{0}^{3}}{r^{2}} \sin \varphi\left(1+\alpha \sin \frac{z}{z_{0}}\right)$.

In the next step, $V_{z}$ is derived from Eqs. (11) or (12), while Eq. (13) gives the relationship between $V_{r}$ and $V_{\varphi}$. In other words, the last two components are not determined directly by the scalar function $F$ if $\boldsymbol{B}$ is already known. For this we need to solve the system of Eqs. (10) with respect to $\boldsymbol{V}$. The coefficient matrix of this system is given by

$\left(\begin{array}{rrr}0 & B_{z} & -B_{\varphi} \\ -B_{z} & 0 & B_{r} \\ B_{\varphi} & -B_{r} & 0\end{array}\right)$

This coefficient matrix has rank 2, and, consequently, we have in fact only two independent equations in the system (10) for the three unknowns, $V_{r}, V_{\varphi}$, and $V_{z}$. The third equation, Eq. (13), is also an additional condition for the choice of the function $F$. From Eq. (12) it follows that

$V_{z}=V_{0}\left(1+\alpha \sin \frac{z}{z_{0}}\right)$.

And from Eq. (13) we obtain

$V_{r}=-\frac{r^{3}-r_{0}^{3}}{r^{3}+2 r_{0}^{3}}\left(\alpha V_{0} \frac{r}{z_{0}} \cos \frac{z}{z_{0}}+V_{\varphi} \frac{\cos \varphi}{\sin \varphi}\right)$. 


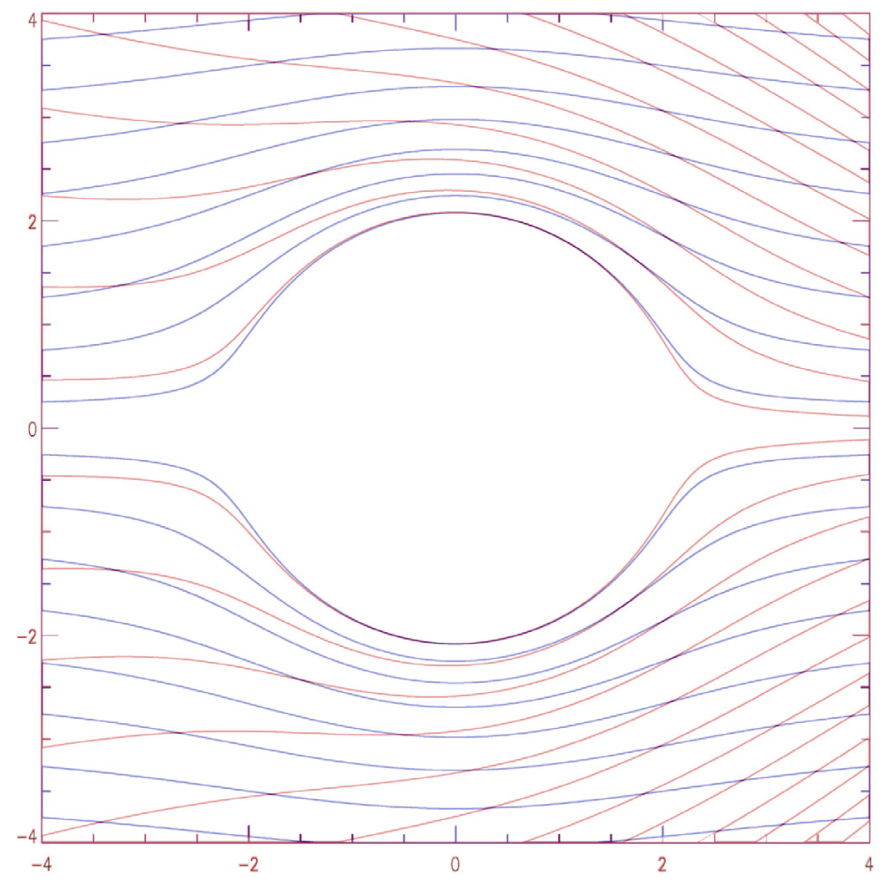

Fig. 1. Magnetic field lines (blue), and streamlines (red), around a cylindrical magnetic cloud.

To have a finite $V_{r}$ at $\varphi= \pm \pi$, it is necessary to take $V_{\varphi}=$ $V_{1} \sin \varphi$, where $V_{1}$ is an arbitrary function of $r, \varphi$, and $z$. For example, we can set

$V_{\varphi}=V_{1}^{0}\left(1+\frac{r}{r_{0}}\right) \sin \varphi$

One can see that Eq. (23) can only be used for

$r<\frac{z_{0}}{\alpha} \frac{1}{\cos \frac{z}{z_{0}}}$

but this restriction is quite acceptable since it can always be satisfied by an appropriate selection of $z_{0}, \alpha$, and $z$, suitable for describing the considered real objects.

We consider a cylindrical magnetic cloud. The magnetic field lines are given by Eqs. (6), (7) and the velocity streamlines obtained from Eqs. (23), (24). The magnetic field lines (in blue) and the streamlines (in red) are both shown in Fig. 1. In Fig. 2 one can see a contour plot of the angle between $\boldsymbol{V}$ and $\boldsymbol{B}$. Contours of the velocity and the magnetic field magnitudes are shown in Figs. 3 and 4, respectively.

\subsection{The general case $\left(B_{x, y, z} \neq 0\right)$}

Let us now also consider the general case, with $B_{x, y, z} \neq 0$; i.e. all three components of the magnetic field are different from zero. Assume that the magnetic field is given by

$$
\begin{aligned}
& B_{r}=B_{0}\left(1-\frac{r_{0}^{3}}{r^{3}}\right) \frac{\cos \varphi}{1+\frac{z^{2}}{z_{0}^{2}}} \\
& B_{\varphi}=-\frac{B_{0}}{2}\left(1+2 \frac{r_{0}^{3}}{r^{3}}\right) \frac{\sin \varphi}{1+\frac{z^{2}}{z_{0}^{2}}}, \\
& B_{z}=-\frac{B_{0}}{2 r}\left(1+2 \frac{r_{0}^{3}}{r^{3}}\right)\left[z_{1}+z_{0} \arctan \frac{z}{z_{0}}\right] \cos \varphi .
\end{aligned}
$$

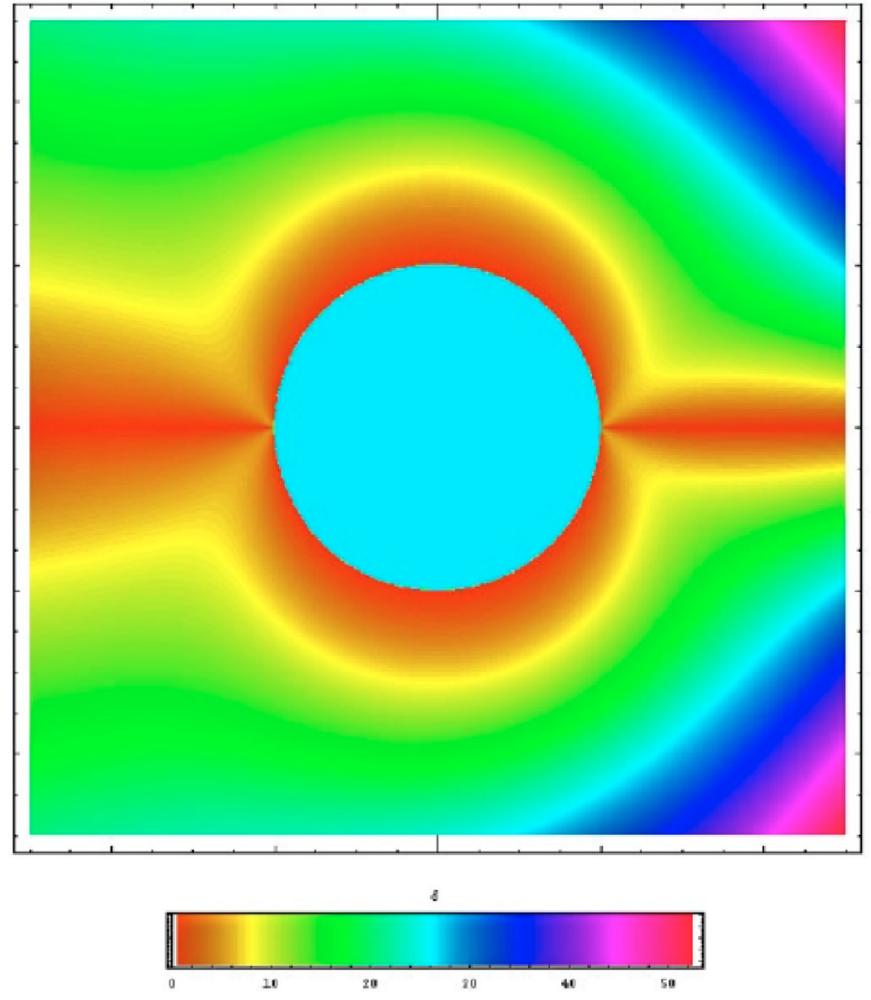

Fig. 2. The angle between the magnetic field and the velocity around the body for the same case as shown in Fig. 1.

From Eq. (10) it is obvious that we have

$\boldsymbol{B} \cdot \nabla F=0$,

and also

$\boldsymbol{V} \cdot \nabla F=0$.

Again applying the method of separation of variables and assuming $F=R(r) \Phi(\varphi) Z(z) B_{0} V_{0}$ (with both $R$, $\Phi$, and $Z$ dimensionless), Eq. (28) can be rewritten in the form:

$$
\begin{aligned}
\frac{r\left(r^{3}-r_{0}^{3}\right)}{r^{3}+2 r_{0}^{3}} & \frac{1}{R} \frac{\mathrm{d} R}{\mathrm{~d} r}-\frac{\sin \varphi}{2 \cos \varphi} \frac{1}{\Phi} \frac{\mathrm{d} \Phi}{\mathrm{d} \varphi} \\
& -\left(z_{1}+z_{0} \arctan \frac{z}{z_{0}}\right)\left(1+\frac{z^{2}}{z_{0}^{2}}\right) \frac{1}{2 Z} \frac{\mathrm{d} Z}{\mathrm{~d} z}=0 .
\end{aligned}
$$

Since the variables are separated in Eq. (30), each of the terms should be equal to a (positive) constant. We again choose $k$ as the constant related to the third term of Eq. (30), so that we have $\left(z_{1}+z_{0} \arctan \frac{z}{z_{0}}\right)\left(1+\frac{z^{2}}{z_{0}^{2}}\right) \frac{1}{2 Z} \frac{\mathrm{d} Z}{\mathrm{~d} z}=k$.

Thus, rewriting this equation in a more standard form, we get

$\frac{1}{Z} \frac{\mathrm{d} Z}{\mathrm{~d} z}=\frac{2 k}{\left(z_{1}+z_{0} \arctan \frac{z}{z_{0}}\right)\left(1+\frac{z^{2}}{z_{0}^{2}}\right)}$.

Similarly, for the $\varphi$-dependent part, by putting the second term in Eq. (30) equal to a (positive) constant $l$ we obtain

$\frac{1}{\Phi} \frac{\mathrm{d} \Phi}{\mathrm{d} \varphi}=2 l \frac{\cos \varphi}{\sin \varphi}$. 

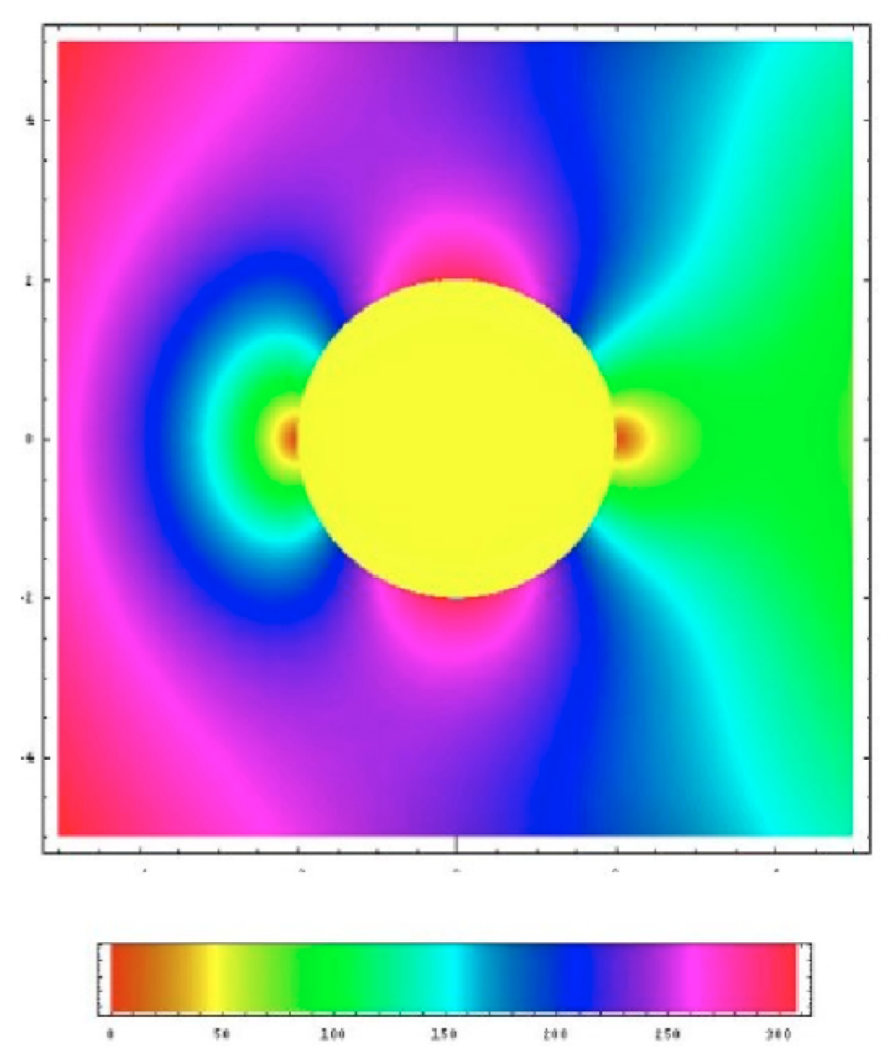

Fig. 3. Contour plot of the velocity magnitude for the same case as shown in Fig. 1.

Clearly, the first term in Eq. (30) should then be equal to $k+l$ so that we get as equation for $R(r)$

$\frac{1}{R} \frac{\mathrm{d} R}{\mathrm{~d} r}=\frac{k+l}{r} \frac{r^{3}+2 r_{0}^{3}}{r^{3}-r_{0}^{3}}$.

We now choose $k=l=\frac{1}{2}$ (so that $k+l=1$ ). One can see that in Eq. (29), only expressions for $\frac{1}{R} \frac{\partial R}{\partial r}, \frac{1}{\Phi} \frac{\partial \Phi}{\partial \varphi}$, and $\frac{1}{Z} \frac{\partial Z}{\partial z}$ are needed for determining $\boldsymbol{V}$. We have found these expressions in Eqs. (33), (32), and (31), respectively. Hence, substituting these expressions in Eq. (29), we obtain the following equation for $\boldsymbol{V}$ :

$$
\begin{aligned}
V_{r}\left(\frac{1}{r} \frac{r^{3}+2 r_{0}^{3}}{r^{3}-r_{0}^{3}}\right)+ & V_{\varphi}\left(\frac{\cos \varphi}{\sin \varphi}\right) \\
& +V_{z} \frac{1}{\left(z_{1}+z_{0} \arctan \frac{z}{z_{0}}\right)\left(1+\frac{z^{2}}{z_{0}^{2}}\right)}=0 .
\end{aligned}
$$

The choice of $V_{\varphi}$ is limited to the set of functions yielding finite values of $V_{\varphi} \frac{1}{\Phi} \frac{\mathrm{d} \Phi}{\mathrm{d} \varphi}$ for all $\varphi$. On the other hand, there are no extra requirements for the choice of $V_{z}$, if $\left|z_{1}\right|>\frac{\pi}{2}\left|z_{0}\right|$. Otherwise, the expression for $V_{z}$ should contain $z_{1}+z_{0} \arctan \frac{z}{z_{0}}$. One can see that the following velocity distribution can satisfy Eq. (34):

$$
\begin{aligned}
V_{r}= & -\frac{r^{3}-r_{0}^{3}}{r^{3}+2 r_{0}^{3}}\left\{V_{1}\left(1+\frac{r_{0}}{r}\right) \cos \varphi\right. \\
& \left.+V_{0} r_{0} \frac{1+\alpha \sin \frac{z}{z_{0}}}{\left(z_{1}+z_{0} \arctan \frac{z}{z_{0}}\right)\left(1+\frac{z^{2}}{z_{0}^{2}}\right)}\right\}, \\
V_{\varphi}= & V_{1}\left(1+\frac{r_{0}}{r}\right) \sin \varphi,
\end{aligned}
$$

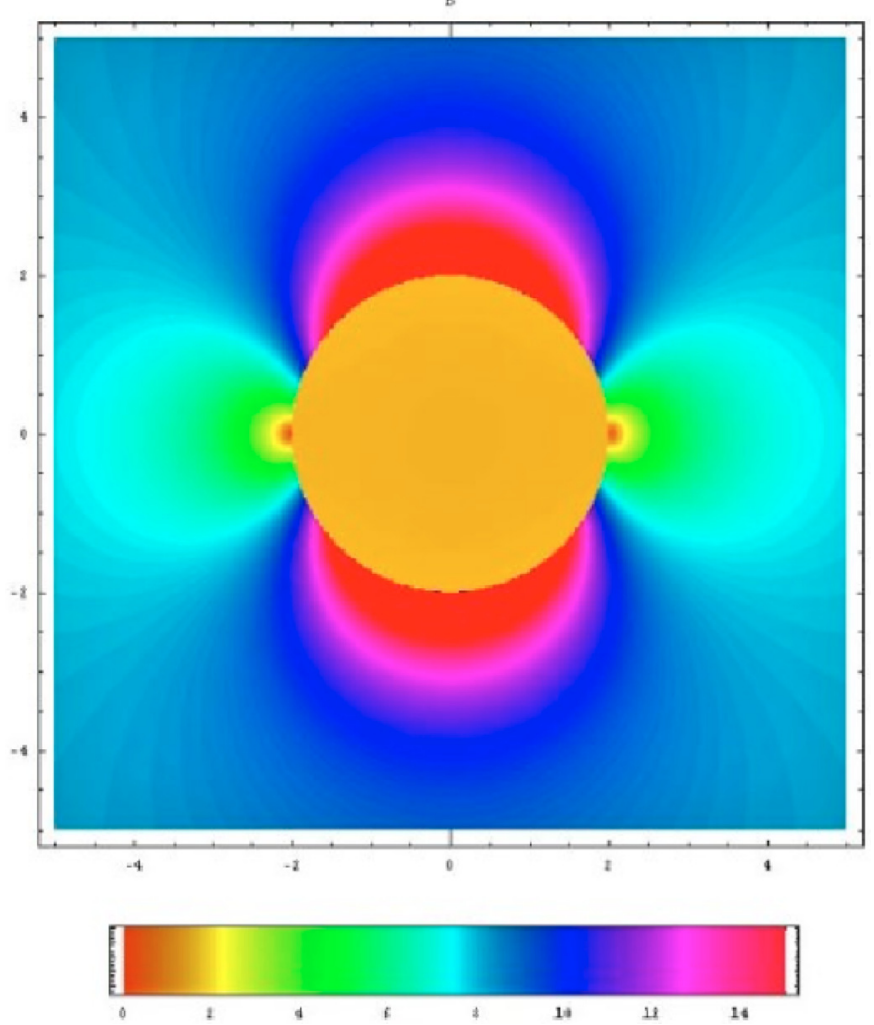

Fig. 4. Magnetic field magnitude contours for the same case as shown in Fig. 1.

$$
V_{z}=V_{0} \frac{r_{0}}{r}\left(1+\alpha \sin \frac{z}{z_{0}}\right) \text {. }
$$

At infinity, the velocity distribution given in Eqs. (35)-(37) approaches undisturbed uniform values, i.e. $V_{r}=-V_{1} \cos \varphi$, $V_{\varphi}=V_{1} \sin \varphi$, and $V_{z}=0$, if we set $z_{1}>V_{0} r_{0} / V_{1}$. In this respect, the solution with $B_{z} \neq 0$ gives a more general solution, which can be extended as far from the tube as needed for the task being considered. While in the two-dimensional case (Fig. 3), Eqs. (22)-(24) are restricted by $r<z_{0} / \alpha$, and these equations are in principle applicable only for a description of plasma flows with bow shock waves or other discontinuities.

\subsection{Determination of the plasma density and pressure}

Having determined the magnetic field and the velocity, one can use the formulas expressing the plasma density $\varrho$ and the thermal pressure $P$ as explicit functions of only $\boldsymbol{V}$ and $\boldsymbol{B}$, which directly follow from (1)-(5) (see Romashets et al. 2005)

$\varrho=-\frac{1}{8 \pi} \frac{\boldsymbol{V} \cdot \nabla \frac{\boldsymbol{V} \cdot \boldsymbol{B} \times(\nabla \times \boldsymbol{B})}{\nabla \cdot \boldsymbol{V}}+\gamma \boldsymbol{V} \cdot \boldsymbol{B} \times(\nabla \times \boldsymbol{B})}{\boldsymbol{V} \cdot \nabla \frac{\boldsymbol{V} \cdot(\boldsymbol{V} \cdot \nabla) \boldsymbol{V}}{\nabla \cdot \boldsymbol{V}}+(\gamma-1) \boldsymbol{V} \cdot(\boldsymbol{V} \cdot \nabla) \boldsymbol{V}}$,

and

$$
\begin{aligned}
P= & -\frac{1}{8 \pi} \frac{\boldsymbol{V} \cdot(\boldsymbol{V} \cdot \nabla) \boldsymbol{V}}{\gamma \nabla \cdot \boldsymbol{V}} \\
& \times \frac{\boldsymbol{V} \cdot \nabla \frac{\boldsymbol{V} \cdot \boldsymbol{B} \times(\nabla \times \boldsymbol{B})}{\nabla \cdot \boldsymbol{V}}+\gamma \boldsymbol{V} \cdot \boldsymbol{B} \times(\nabla \times \boldsymbol{B})}{\boldsymbol{V} \cdot \nabla \frac{\boldsymbol{V} \cdot(\boldsymbol{V} \cdot \nabla) \boldsymbol{V}}{\nabla \cdot \boldsymbol{V}}+(\gamma-1) \boldsymbol{V} \cdot(\boldsymbol{V} \cdot \nabla) \boldsymbol{V}} \\
& +\frac{\boldsymbol{V} \cdot \boldsymbol{B} \times(\nabla \times \boldsymbol{B})}{8 \pi \gamma \nabla \cdot \boldsymbol{V}} .
\end{aligned}
$$


That is, if there is steady-state plasma flow, described by Eqs. (1)-(5), then its density and pressure distributions are given by these equations, as we demonstrate below. The formulas (38) and (39) are applicable only if $\boldsymbol{B} \neq 0$ or $\nabla \boldsymbol{B} \neq 0^{1}$. In fact, Eqs. (38) and (39) only yield a first approximation for plasma density and plasma pressure that are to be used in an iteration procedure to obtain the solution for the entire system (1)-(5). On the other hand, for those $\boldsymbol{B}$ and $\boldsymbol{V}$ that are solutions of the considered problem, Eqs. (38) and (39) give correct values and do satisfy the remaining two scalar and one vector equations, Eqs. (3)-(5). As one can see from the derivation of these formulas, we obtain from Eq. (3)

$\rho \nabla \cdot \boldsymbol{V}=-\boldsymbol{V} \cdot \nabla \rho$

while Eq. (5) yields

$\boldsymbol{V} \cdot \frac{\nabla P}{\rho^{\gamma}}-\gamma \frac{P \boldsymbol{V} \cdot \nabla \rho}{\rho^{\gamma+1}}=0$.

Upon substituting the term $\boldsymbol{V} \cdot \nabla \rho$ from the RHS of Eq. (40) into Eq. (41) we obtain

$\boldsymbol{V} \cdot \nabla P=-\gamma P(\nabla \cdot \boldsymbol{V})$.

Multiplying Eq. (4) by a vector $\boldsymbol{V}$ and substituting $\boldsymbol{V} \cdot \nabla P$ from Eq. (42) in the resulting equation yields

$\boldsymbol{V} \cdot \rho(\boldsymbol{V} \cdot \nabla) \boldsymbol{V}-\gamma P(\nabla \cdot \boldsymbol{V})+\boldsymbol{V} \cdot \nabla\left(\frac{\boldsymbol{B}^{2}}{8 \pi}\right)=V \frac{(\boldsymbol{B} \cdot \nabla) \boldsymbol{B}}{4 \pi}$.

By expressing $P$ as a function of $\rho, \boldsymbol{V}$, and $\boldsymbol{B}$, we can write this in the form

$$
\begin{aligned}
P & =\frac{1}{\gamma(\nabla \cdot \boldsymbol{V})}\left[\rho \boldsymbol{V} \cdot(\boldsymbol{V} \cdot \nabla) \boldsymbol{V}+\boldsymbol{V} \cdot \nabla\left(\frac{\boldsymbol{B}^{2}}{8 \pi}\right)-\frac{\boldsymbol{V} \cdot(\boldsymbol{B} \cdot \nabla) \boldsymbol{B}}{4 \pi}\right] \\
& =\rho S_{1}+S_{2} .
\end{aligned}
$$

Here, term $S_{1}$ is defined as $S_{1}=\frac{\boldsymbol{V} \cdot(\boldsymbol{V} \cdot \nabla) \boldsymbol{V}}{\gamma \nabla \cdot \boldsymbol{V}}$, and $S_{2}$ is defined as $S_{2}=\frac{\boldsymbol{V} \cdot \nabla\left(\boldsymbol{B}^{2}\right)-2 \boldsymbol{V} \cdot(\boldsymbol{B} \cdot \nabla) \boldsymbol{B}}{8 \pi \gamma \nabla \cdot \boldsymbol{V}}=\frac{\boldsymbol{V} \cdot \boldsymbol{B} \times(\nabla \times \boldsymbol{B})}{8 \pi \gamma \nabla \cdot \boldsymbol{V}}$.

In order to find $\rho$, we now substitute the expression for $P$ from Eq. (44) in Eq. (42). This yields

$\boldsymbol{V} \cdot \nabla\left(\frac{S_{1}}{\rho^{\gamma-1}}+\frac{S_{2}}{\rho^{\gamma}}\right)=\frac{\boldsymbol{V} \cdot \nabla S_{1}}{\rho^{\gamma-1}}-\frac{(\gamma-1) S_{2}}{\rho^{\gamma}} \boldsymbol{V} \cdot \nabla \rho=0$.

The latter equation can be rewritten as

$\rho=\frac{-\left[\boldsymbol{V} \cdot \nabla S_{2}+\gamma S_{2}(\nabla \cdot \boldsymbol{V})\right]}{\left[\boldsymbol{V} \cdot \nabla S_{1}+(\gamma-1) S_{1}(\nabla \cdot \boldsymbol{V})\right]}$.

Hence, Eq. (38) directly follows from Eq. (46), and then Eq. (39) follows from Eq. (44).

The iteration process can be organised in the following manner. First, we take a first approximation of the magnetic field that should obviously satisfy Eq. (1), as well as the proper boundary conditions. In our case, $B_{r}$ has to vanish on the surface of the tube (i.e. at $r=r_{0}$ ) and, in the limit $r \rightarrow \infty$, the magnetic field should behave like the pre-existing magnetic field. Because Eq. (1) is scalar, we can set $B_{r}$ in the beginning, while $B_{\varphi}$ remains free, and $B_{z}$ is given by the solenoidal condition; this is

${ }_{1}$ As a matter of fact, if $\boldsymbol{B}$ and/or $\nabla \boldsymbol{B}$ are zero, then $S_{2}=0\left(S_{2}\right.$ is defined after Eq. (44)). Once $S_{2}$ is zero, it is impossible to solve Eq. (45) in the way shown in Eq. (46). This is why $S_{2}$ should be non-zero. Hence, if there is no magnetic field, $\boldsymbol{B}=0$, these formulas are not applicable. the meaning of Eq. (1). For example, if at infinity $B_{r}$ should behave as $B_{0} \cos \varphi$ (as in the case of the filed distribution given by Eqs. (6)-(8), we can set $B_{r}=B_{0}(1-f(r)) \cos \varphi$, with $f\left(r_{0}\right)=1$ and $\lim _{r \rightarrow \infty} f(r)=0$. The velocity field is taken again from Eq. (2), like in Eqs. (22)-(24). But, as said above, there is one degree of freedom in the choice of $\boldsymbol{V}$. In other words, if we have set $V_{r}$ and $V_{\varphi}$, then $V_{z}$ is still a free function, to be used for satisfying the remaining equations. From Eq. (3) we then obtain an approximation of $\rho$ in the following way:

$\rho=\frac{\boldsymbol{V} \cdot(\nabla \times \boldsymbol{H})}{\boldsymbol{V}^{2}}$.

Equation (47) is derived in the following way. From Eq. (3) it follows that $\rho \boldsymbol{V}$ can be represented in the form $\nabla \times \boldsymbol{H}$, where $\boldsymbol{H}\left(H_{r}, H_{\varphi}, H_{z}\right)$ is an arbitrary vector apart from the fact that it should satisfy the inner and outer boundary conditions for $\rho$, as well as for $\boldsymbol{V}$. The conditions are that both $\rho$ and $\boldsymbol{V}$ should be finite in the whole volume, even on the surface; and at large distances from the body, they approach undisturbed pre-existing values. The radial component of the velocity should be zero at $r=r_{0}$. From Eq. (4) we have

$\nabla P=-\frac{1}{8 \pi} \boldsymbol{B} \times(\nabla \times \boldsymbol{B})-\rho(\boldsymbol{V} \cdot \nabla) \boldsymbol{V}$.

The vector $\boldsymbol{H}\left(H_{r}, H_{\varphi}, H_{z}\right)$ should also satisfy Eq. (5); i.e. we should have

$P=-\frac{\frac{V \cdot(\nabla \times \boldsymbol{H})}{\boldsymbol{V}^{2}} \boldsymbol{V} \cdot\left(\frac{1}{8 \pi} \boldsymbol{B} \times(\nabla \times \boldsymbol{B})+\frac{\boldsymbol{V} \cdot(\nabla \times \boldsymbol{H})}{\boldsymbol{V}^{2}}(\boldsymbol{V} \cdot \nabla) \boldsymbol{V}\right)}{\boldsymbol{V} \cdot \nabla \frac{\boldsymbol{V} \cdot(\nabla \times \boldsymbol{H})}{\boldsymbol{V}^{2}}}$.

Note that formulas (47) and (49) for $\rho$ and $P$, respectively, are very complicated. It thus remains very difficult to calculate these quantities in this way. Since both $B$ and $V$ have their maximums on the boundary of about four times the undisturbed values, it seems that both $\rho$ and $P$ should give a maximum increase at the tube, of the order of 2 to 4 times the undisturbed values at infinity. At infinity, $P_{0}$ and $\rho_{0}$ are the pre-defined uniform values.

Equation (5) is satisfied by proper selection of $H_{r}, H_{\varphi}, H_{z}$, $V_{z}$, and $B_{\varphi}$, while the first four equations (i.e. Eqs. (1)-(4)) are already satisfied. We thus have five scalar functions for satisfying five scalar equations given by $(3)-(5)$. Note that $P$ should also be finite on the inner boundary and coincide with the value of the pre-existing $P$ at infinity. If this is not the case, then we return to Eq. (1) and change the setting of the function $f(r)$, and finally repeat the calculation cycle. In total, there are nine scalar equations in the system (1)-(5), since it contains two vector equations (with three components) and three scalar equations, for eight unknowns. However, there is no contradiction here due to the type of Eq. (2), which is in fact only equivalent to two scalar equations, because the third one is a linear combination of the first and the second ones (see the matrix given after Eq. (21)).

An alternative approach can be to eliminate $B_{z}$ with Eq. (1) and then to eliminate $B_{\varphi}$ end $V_{z}$ by means of Eq. (2) using $\boldsymbol{B} \cdot \nabla F=0$ and $\boldsymbol{V} \cdot \nabla F=0$, which follow from Eq. (10). Using Eqs. (38) and (39) for eliminating $\rho$ and $P$, respectively, we are then left with one vector equation (Eq. (4)) for three unknowns, i.e. $B_{r}, V_{\varphi}$, and $V_{r}$. The conservation of mass and energy are guaranteed by formulas (38) and (39), as can be seen in the derivation above. The boundary conditions can be satisfied by proper selection of the function $F$ and the integration constant coming from Eq. (1). 


\section{Discussion}

The difficulties describing a plasma flow around a cylindrical obstacle have been discussed earlier (Farrugia et al. 1988). If a cylinder, with its axis along the $z$-direction and with a superconducting surface, is placed inside a region where the magnetic field was planar, i.e. with only $r$-and $\varphi$-components, then the new disturbed field is also planar. Moreover, so-called stagnation points arise where the magnetic field is disappearing. In this case, the stagnation points lie on two lines along the $z$-axis. The new disturbed field is more or less similar to the magnetic field specified by Eqs. (6)-(8).

On the other hand, the velocity field also remains planar, if it was before the cylinder was put into the flow. With both $V_{z}=$ 0 and $B_{z}=0$ and if there is no dependence on $z$, Eq. (9) is equivalent to

$\frac{\partial}{\partial r}\left(V_{r} B_{\varphi}-V_{\varphi} B_{r}\right)=0$

and

$\frac{\partial}{\partial \varphi}\left(V_{r} B_{\varphi}-V_{\varphi} B_{r}\right)=0$

This simply means that

$V_{r} B_{\varphi}-V_{\varphi} B_{r}=$ const.

On the other hand, since there are null points where both $B_{r}$ and $B_{\varphi}$ vanish and the velocity is finite, so that const $=0$ in Eq. (52), then

$\frac{V_{r}}{V_{\varphi}}=\frac{B_{r}}{B_{\varphi}}$.

This means that both vectors, $\boldsymbol{V}$ and $\boldsymbol{B}$, coincide everywhere. This difficulty encountered in the calculation was discussed in Erkaev et al. (1995). The only possibility to construct the solution without field and stream penetration through the surface of the cloud was to assume $\boldsymbol{V} \| \boldsymbol{B}$, in two dimensional calculations. In this respect, Erkaev et al. (1995) considered the case where $\boldsymbol{V}$ has nonzero values at $r=r_{0}$, to have $\boldsymbol{V} \nVdash \boldsymbol{B}$. We showed here, in this paper, that the difficulty can be solved in three dimensions. Once there is a non-zero $V_{z}$ available, which means that the tube axis is inclined with respect to the incoming plasma flow, we constructed finite distributions of the velocity and the magnetic field without penetration at the cloud's boundary. Obviously, one has to consider the full three-dimensional problem to treat the $\boldsymbol{V} \nVdash \boldsymbol{B}$ case, which is usually observed in the solar wind.

We have presented above a few sample solutions of this type. We did not discuss the magnetic field and the plasma distribution inside the object, as this is not the subject of the present paper. In short, the field there is expected to be force-free, see, for example, Romashets \& Vandas (2005). The temperature and density are usually lower inside that magnetic cloud than in the ambient medium. Solutions for the magnetic field and plasma distribution inside should also satisfy boundary conditions at $r=r_{0}$, meaning that on the surface we then have a tangential discontinuity. For the internal solution $\boldsymbol{B}_{r}=0$ at $r=r_{0}$, And all parameters are finite inside, i.e. for $r \leq r_{0}$. Formulas (38) and (39 follow directly, i.e. without any additional assumptions, from the system of MHD equations in the steady form, i.e. Eqs. (1)-(5).

Since both Eqs. (38) and (39) contain the term $\nabla \cdot \boldsymbol{V}$ in their denominators, we should confine their use to regions where $\nabla \cdot \boldsymbol{V} \neq 0$ or, in other words, to compressible/expandable plasmas. For incompressible plasmas, the system (1)-(5), where Eq. (3) is replaced by

$\nabla \cdot V=0$

must still be solved.

\section{Conclusions}

In the present paper we have determined the plasma parameters of a steady plasma flow around a cylindrical object, modelling a CME flux rope in the solar corona and the solar wind. We first considered the simpler case with $B_{z}=0$. We used the stationary form of the MHD equations and first chose a magnetic field configuration satisfying the boundary conditions at the flux rope surface and at infinity, as well as the condition of finiteness. Next, the corresponding velocity distribution was determined by making use of the frozen-in condition that is valid in ideal MHD and thus, to a large extent, also in the nearly-perfectly conducting solar corona and solar wind. Finally, the corresponding plasma density and plasma pressure are determined completely analytically. This procedure was then repeated for the more general case with all three components of the magnetic field that are different from zero. The calculations then become somewhat more involved, but the procedure again yields a satisfactory result.

The procedure, as proposed and applied in the present paper, contains a new method for calculating the plasma parameters, i.e. the plasma density and the thermal pressure around the body embedded in the solar corona or the solar wind. The maximum increase in velocity and magnetic field is about 3-4 times in the layers around the cloud. It seems that some MHD models (Riley et al. 2004; Odstrcil et al. 2004; Manchester et al. 2004) give similar results as this analytical approach.

It is clear that this procedure may not be able to describe all the peculiarities in the dynamics of the flow around a magnetic cloud, but the calculations are substantially faster than a full three-dimensional MHD simulation. Even with extremely powerful parallel computers, such MHD simulations take longer than the real time evolution of the cloud, which is a problem if one wants to predict the geo-effectiveness of the CME and its arrival time and impact angle. Moreover, the satisfaction of the boundary conditions at infinity becomes clearer in the simpler approach presented here.

The flows patterns obtained with this model can help shed light on the debate as to whether ICME cross-sections are distorted from convex- to concave-outward structures by the ambient solar wind (Odstrcil et al. 2004; Owens 2006). If the IMF is mostly radial, then the maximum of the ambient magnetic field is reached on the cloud's sides, and this leads to an elongation of the cloud along the Sun-Earth line. If the IMF has a significant non-radial component, however, tear-drop type structures most likely will be formed.

There is some analogy between the flow around ICMEs and the flow around the Earth's magnetosphere. The major difference, however, is that the flow around the magnetosphere is always supersonic, while the flows past ICMEs can be both supersonic or subsonic, without formation of a bow shock, i.e. when the difference between the cloud velocity and the velocity of the ambient solar wind is lower than the local Alfvén speed, which is typically of the order of $70 \mathrm{~km} \mathrm{~s}^{-1}$.

To apply our model to data analysis, one should first learn (e.g. from minimum variance methods) the major parameters of the cloud, i.e. its radius, the orientation of its axis, and the magnitude of the magnetic field at the axis. Then, the velocity at 
infinity is set to be along the Sun-Earth line, and the magnetic field at infinity is assumed to be uniform, with the direction and magnitude of the pre-existing quiet solar wind. In this model, the velocity, magnetic field, plasma pressure, and density are constructed in such a way that both the radial velocity and the radial magnetic field vanish at the boundary, i.e. by the selection of the free parameters. However, it should be noticed that the choice of the free parameters, for example $V_{0}$ and $V_{1}$, does not affect the physics of the deflected flows. On the other hand, there are many restrictions on these choices, based on the knowledge of the typical values of radial and non-radial components of the solar wind, density, and magnetic field in the sheath region of the clouds.

Acknowledgements. The authors wish to express sincere thanks to many colleagues for useful discussions. The work was supported by BELSPO, RFBR grant 06-05-64500, and the EU/INTAS grant 03-51-6206. These results were obtained in the framework of the projects GOA/2004/01 (K.U. Leuven), G.0304.07 (FWO-Vlaanderen), C 90203, and C 90196 (ESA Prodex 8). E.R. was supported by a BELSPO fellowship.

\section{References}

Cargill, P. J. 2004, Sol. Phys., 221, 135

Chane, E., van der Holst, B., Jacobs, C., Poedts, S., \& Kimpe, D. 2006, AA, 447, 727
Detman, T. R., Dryer, M., Yeh, T., Han, S. M., \& Wu, S. T. 1991, JGR, 96, 9531 Erkaev, N. V., Farrugia, C. J., Biernat, H. K., Burlaga, L. F., \& Bachmaier, G. A. 1995, JGR, 100, 19919

Farrugia, C. J., Elphic, R. C., Southwood, D. J., \& Cowley, S. W. H. 1987, PSS, 35,227

Farrugia, C. J., Fitzenreiter, R. J., Burlaga, L. F., et al. 1994. ASR, 14(7), 105

Gosling, J. T., \& McComas, D. J. 1987, GRL, 14, 355

Manchester, W. B., Gombosi, T. I., Roussev, I., et al. 2004, JGR, 109

McComas, D. J., Gosling, J. T., Winterhalter, D., \& Smith, E. J. 1988, JGR, 93, 2519

Odstrcil, D., \& Pizzo, V. J. 1999, JGR, 104, 28,225

Odstrcil, D., Riley, P., Zhao, X. P.2004, JGR, 109, A02116

Owens, M. J. 2006, JGR, 111, A12109

Owens, M., \& Cargill, P. 2002, AG, 22, 4397

Owens, M. J., Cargill, P. J., Pagel, C., Siscoe, G. L., \& Crooker, N. U. 2005, JGR, 110

Owens, M. J., Merkin, V. G., \& Riley, P. 2006, JGR, 111, A03104

Riley, P., \& Crooker, N. U. 2004, ApJ, 600, 1035

Riley, P., Linker, J. A., Lionello, R., et al. 2004, JASTP, 66, 1321

Romashets, E. P., \& Vandas, M. 2005, ASR, 35, 2167

Romashets, E. P., Vandas, M., \& Nagatsuma, T. 2005, in Proc. ISSS-7, ed. H. Matsumoto, 403

Vandas, M., \& Romashets, E. 2003, AA, 398, 801

Vršnak, B. 2001, JGR, 106, 25291 